\title{
New insights into the secretory functions of brown adipose tissue
}

\author{
Joan Villarroya, Rubén Cereijo, Aleix Gavaldà-Navarro, Marion Peyrou, Marta Giralt and Francesc Villarroya \\ Departament de Bioquímica i Biomedicina Molecular and Institut de Biomedicina (IBUB), Universitat de Barcelona, Barcelona, Catalonia, Spain \\ CIBER Fisiopatología de la Obesidad y Nutrición, Barcelona, Catalonia, Spain
}

Correspondence should be addressed to F Villarroya: fvillarroya@ub.edu

\begin{abstract}
In recent years, an important secretory role of brown adipose tissue (BAT) has emerged, which is consistent, to some extent, with the earlier recognition of the important secretory role of white fat. The so-called brown adipokines or 'batokines' may play an autocrine role, which may either be positive or negative, in the thermogenic function of brown adipocytes. Additionally, there is a growing recognition of the signalling molecules released by brown adipocytes that target sympathetic nerve endings (such as neuregulin-4 and S100b protein), vascular cells (e.g., bone morphogenetic protein-8b), and immune cells (e.g., C-X-C motif chemokine ligand-14) to promote the tissue remodelling associated with the adaptive BAT recruitment in response to thermogenic stimuli. Moreover, existing indications of an endocrine role of BAT are being confirmed through the release of brown adipokines acting on other distant tissues and organs; a recent example is the recognition that BAT-secreted fibroblast growth factor-21 and myostatin target the heart and skeletal muscle, respectively. The application of proteomics technologies is aiding the identification of new members of the brown adipocyte secretome, such as the extracellular matrix or complement system components. In summary, BAT can no longer be considered a mere producer of heat in response to environment or dietary challenges; it is also an active secretory tissue releasing brown adipokines with a relevant local and systemic action. The identification of the major brown adipokines and their roles is highly important for the discovery of novel candidates useful in formulating intervention strategies for metabolic diseases.
\end{abstract}

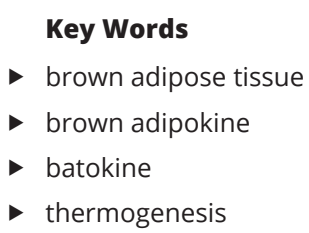

- brown adipose tissue

- brown adipokine

thermogenesis

\section{Introduction: the evolving research on the BAT secretome}

In recent years, extensive research has been conducted to elucidate the secretory role of brown adipose tissue (BAT), including its endocrine role. In certain ways, the history of this research has followed a parallel and delayed path relative to the research of the white adipose tissue (WAT) secretome. For decades, the biological function of WAT was considered to be restricted to fat storage and the release of metabolic foodstuffs when needed; similarly, BAT has been considered to essentially play a role in energy expenditure to support non-shivering thermogenesis. We know that, in brown adipocytes, there is a regulated uncoupling of the mitochondrial respiratory chain relative to ATP synthesis in order to produce heat, owing to the unique presence of the uncoupling protein-1 (UCP1) in brown adipocyte mitochondria, and this makes the brown adipocytes specialised for the consumption of metabolic foodstuffs 
to produce heat. Currently, we also have a comprehensive understanding of the physiological (mainly sympathetic nervous system-mediated) mechanisms of the regulation of BAT as a heat-producing system with regard to thermic stimuli in the environment, and possibly, to dietary stimuli (Cannon \& Nedergaard 2004). Several decades ago, mostly fuelled by the discovery of leptin, WAT had begun to be recognised as a source of regulatory proteins targeting distinct organs and tissues. The term 'adipokine' was adopted to describe the myriad of molecules secreted by white fat that have been described to date (Blüher \& Mantzoros 2015). However, the potential secretory role of BAT has been intensely investigated only much more recently. Possibly, the low expression of many 'white' adipokines, such as leptin, in brown fat and the low expression of pro-inflammatory cytokines known to be released by WAT (e.g. tumour necrosis factor- $\alpha$; TNF $\alpha$ ) (Cannon \& Nedergaard 2004) may have also contributed to a somewhat historically neglected research on the secretory role of BAT. In principle, a rather distinct pattern of factors secreted by BAT, relative to the case for WAT, is expected; given the distinct (and even opposite) role of BAT (energy expenditure) and WAT (energy storage) in energy metabolism, it is unlikely that their secretomes are similar.

Research on the secretory role of BAT has been driven by several observations: first, the much more intense systemic phenotype in mouse models with genetically targeted ablation of BAT (e.g. UCP-DTA mice) relative to those with just thermogenic impairment of BAT (e.g. UCP1null mice) (Lowell et al. 1993); second, the widespread observations that the experimental transplantation of small amounts of BAT lead to relevant effects on systemic metabolism, mainly the improvement in glucose homeostasis and even reduced weight gain, which are hardly explainable by the intrinsic thermogenic activity of the transplant (Villarroya \& Giralt 2015, Villarroya et al. 2017a); third, the identification of genes encoding secreted proteins as a part of the pattern of expression of genes regulated in the BAT in response to thermogenic stimuli. Hence, research during the recent years has led to an increased interest in identifying BAT-secreted factors, especially those capable of acting on organs at a distance and exerting an endocrine effect.

\section{What are brown adipokines or 'batokines'?}

The term 'brown adipokines' (in some cases also called 'batokines') has some imprecisions in its use, which are, in fact, rather similar to those associated with the use of the general term 'adipokine'. In some contexts, 'brown adipokine' is used to refer to proteins (or even other nonpeptidic types of regulatory factors) released by brown fat 'tissue', whereas others restrict the use of the term to proteins released by the brown adipocyte 'cell type'. This is a biologically relevant distinction, considering the presence of non-brown adipocyte cells in the BAT (e.g. macrophages and other immune cells, vascular cells, nerve endings) which may be relevant sources of secreted factors. To date, no molecule reported to be secreted by BAT has been found to be totally 'brown specific' in the sense that, for example, UCP1 protein expression is. Hence, existing literature often qualifies brown adipokines as secreted factors 'preferentially' released by BAT-versusWAT and, in some cases, compared to other tissues or organs. Moreover, in many research reports, there is also the assumption that a brown adipokine is a molecule that is released intensely by the BAT when the tissue is thermogenically activated; this makes sense when the secretory function of BAT is associated with the induction of its physiological role as a heat-producing tissue. There are also some non-consensual uses of the term 'brown adipokine' related to the chemical nature of the released molecule. Whether the recognition of small non-coding RNAs (e.g. miRNA-99b) (Thomou et al. 2017) as relevant signalling molecules released by BAT and targeting the liver also justifies the use of the term 'brown adipokines' is unclear; the same applies to the identifications of BATsecreted factors of lipidic nature (Lynes et al. 2017) which are expected to be named 'brown lipokines'. Thus, it is clear that for any description of the factors secreted by BAT that are capable of exerting signalling properties, the term 'brown adipokine' would be of limited value without the addition of their cellular source, their regulation in relation to the thermogenic activity of BAT and, of course, their chemical nature. There are several relatively recent review articles that summarise the state-of-the-art knowledge regarding brown adipokines (Villarroya et al. $2017 a, b$, Lee et al. 2019); however, the intense research in this field and growing recognition of novel factors secreted by BAT has aided the identification of novel BATsecreted factors and/or expanded our understanding of the targets and functions of some already known batokines. A systematic description of the currently identified brown adipokines and, in general, bioactive factors (including brown lipokines and miRNAs) released by brown and beige adipocytes is provided in Supplementary Table 1 (see section on supplementary data given at the end of this article). Herein, we review the most recent significant 
advances in the knowledge of the BAT secretome and its importance for autocrine, paracrine, and endocrine signalling.

\section{What does proteomics tell us about the BAT secretome and identification of new batokines?}

Considering the progress in proteomics technology, the direct assessment of the proteins released by brown adipocytes appears as an obvious analytical approach to obtain a thorough knowledge of the brown adipocyte secretome. However, only two recent studies utilising this strategy have been published (Ali Khan et al. 2018, Villarroya et al. 2019), and these report the identification of the set of protein factors whose expression is significantly induced in the culture medium of murine brown adipocytes acutely exposed to cAMP (71 proteins) or to noradrenaline (280 proteins), which are similar, but not identical, thermogenic stimuli for the brown fat cell. These data were remarkably coincident, and 60\% (42 of 71) of the proteins found in the study by Villarroya et al. (2019) were also identified in the study by Ali Khan et al. (2018). Some of these factors were adipokines that have already been known to be secreted by brown adipocytes, such as adiponectin (Hui et al. 2015), angiotensinogen (Campbell \& Habener 1987), and chemerin (Hansen et al. 2014). Other previously identified brown adipokines such as fibroblast growth factor-21 (FGF21), neuregulin-4 (NRG4) or bone morphogenetic protein-8b (BMP8b) (Villarroya et al. 2017a) were not found in either of the two studies, and this may be related to the low concentration ranges of these factors and potential limitations of the proteomics technology.

Interestingly, many members of the brown adipocyte secretome commonly identified in the two aforementioned studies were extracellular matrix (ECM) components or regulators (matricellular proteins). Alterations in the formation of a functional ECM have been reported in WAT-related pathological conditions such as obesity. Physiological WAT expansion requires ECM remodelling; reaching a threshold in such capacity when the WAT is maximally overloaded with fat is considered a significant trigger of obesity-associated pathologies (Crewe et al. 2017, Datta et al. 2018). BAT is a highly plastic tissue, and its long-term thermogenic activation leads to hypertrophic and hyperplastic processes, ultimately resulting in adaptive BAT recruitment (Cannon \& Nedergaard 2004). The identification of ECM components (distinct types of collagens) and matricellular proteins (non-structural proteins present in the ECM and having a regulatory role) as members of the secretome of thermogenically activated brown adipocytes possibly highlights the importance of BAT remodelling when the tissue has to be expanded in response to thermogenic activation. The role of the ECM in BAT has been practically unexplored to date; the recent findings based on proteomics analyses clearly indicate the requirement for further research to explore this novel biological process related to the BAT secretome. Hansen (2014), who analysed the mouse brown adipocyte proteome indirectly through a sequence trap genetic analysis, also reported the identification of several ECM components and matricellular proteins such as epidermal growth factor-containing fibulin-like extracellular matrix protein (EFEMP1), collagen $\alpha-1$ (III) chain (COL3A1), procollagen C-proteinase enhancer protein (PCOLCE) or secreted acidic cysteine-rich glycoprotein (SPARC) as a part of the brown adipocyte secretome in response to noradrenaline.

Proteomics studies have also coincidently identified several components of the complement system in the secretomes of cAMP- and noradrenaline-stimulated brown adipocytes. This was a surprising finding. In addition to complement factor-D (also called adipsin), in fact, one of the first white adipokines to be identified (Cook et al. 1987), other factors such as complement C4-B, complement factor- $\mathrm{B}$, and complement $\mathrm{C} 3$ have been reported to be a part of the brown adipocyte secretome according to the studies by both Villarroya et al. (2019) and Ali Kahn et al. (2018). Further research is warranted to ascertain the role of complement system components in the signalling that originates in brown adipocytes upon thermogenic activation.

Finally, it is worth mentioning that if we compile the data obtained from the two direct proteomics analyses of mouse samples and the indirect sequence trap-based analysis, only the following five proteins are commonly found as thermogenic stimulus-induced batokines: two components of the ECM (COL3A1 and PCOLCE), insulinlike growth factor-binding protein-4 (IGFBP4), follistatinlike protein-1 (FSTL1), and chemerin (also named Rarres2, from 'retinoic acid receptor responder-2') (Fig. 1). These factors warrant further investigation as potential batokines, because to date, only chemerin has been specifically studied as an adipokine released by brown adipocytes (Hansen et al. 2014).

Recently, Deshmukh et al. (2018) have reported a proteomics-based analysis of the brown adipocyte 


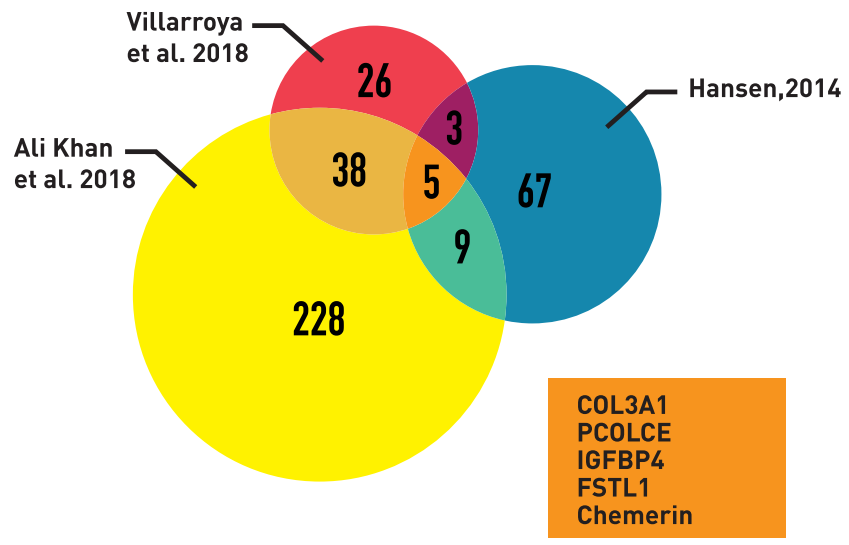

\section{Figure 1}

Venn diagram showing the overlap between the proteins secreted by brown adipocytes in culture in response to CAMP (Villarroya et al. 2018a,b) or noradrenaline (Ali Khan et al. 2018), based on proteomics analysis, and in response to noradrenaline, based on the signal sequence trap technique (Hansen 2014). The numbers of secreted proteins found in the different studies are shown in the intersections. The box indicates the five proteins secreted by thermogenically stimulated brown adipocytes that were commonly identified in these three studies: collagen $\alpha-1$ (III) chain (COL3A1), procollagen C-proteinase enhancer protein (PCOLCE), insulin-like growth factor binding protein-4 (IGFBP4), follistatin-like protein-1 (FSTL1), and chemerin.

proteome from human brown adipocytes in response to noradrenaline. ECM components, complement factors and several other proteins reported to be a part of the thermogenically stimulated mouse brown adipocyte secretome were also found in the noradrenaline-stimulated human brown adipocyte secretome (e.g., fibrillin-1, SPARC-like protein 1, progranulin, and transforming growth factor- $\beta$ receptor-3). These authors also propose that ependymin-related protein 1 (EPDR1) may be a novel human brown adipokine acting on the brown fat activity itself and influencing the overall energy expenditure.

\section{The emerging BAT-to-heart signalling based on FGF21}

FGF21 is among the first proposed endocrine signals acting as a batokine, given the strong release of FGF21 by BAT under conditions of thermogenic activation (Hondares et al. 2011). Accordingly, tissues and organs known to be sensitive to FGF21 action (e.g. WAT, brain, pancreas) have been considered as potential targets of the FGF21 secreted from the BAT. The heart is one of these potential target tissues, given the reports indicating a strong cardioprotective effect of FGF21 (Planavila et al. 2013). However, direct evidence for this was lacking until the study by Ruan and his collaborators, who concluded, as a consequence of a study on the adenosine $\mathrm{A} 2 \mathrm{~A}$ receptor in $\mathrm{BAT}$, that the FGF21 released by BAT targets the heart (Ruan et al. 2018). Brown adipocytespecific FGF21 knockout impaired the previously observed effects of adenosine $\mathrm{A} 2 \mathrm{~A}$ receptor agonism in attenuating hypertensive cardiac remodelling. These findings identify an endocrine role of BAT in controlling hypertensive cardiac remodelling though the release of FGF21. However, Thoonen et al. (2015) have reported that UCP1-null mice show exaggerated myocardial injury, fibrosis, and adverse cardiac remodelling, as well as decreased survival, in response to experimental cardiac insults. Transplantation of functional BAT reversed the myocardial injury and increased the survival of mice, suggesting a systemic cardioprotective role of functional BAT. However, it is well known that BAT from UCP-1-null mice expresses much higher levels of FGF21 than wildtype mice and is thus a significant source of circulating FGF21 (Keipert et al. 2015), suggesting that factors other than FGF21 may contribute to the protective actions of BAT on the heart.

Notably, several studies reporting heart alterations, including signs of diabetic cardiomyopathy, in UCP-DTA mice have been published (Duncan et al. 2007, Ilkun et al. 2015). The UCP-DTA mouse model is based on the transgenic expression of the diphtheria toxin A chain (DTA) under the control of the UCP1 gene promoter. These mice have genetically ablated BAT and are prone to diet-induced obesity due to impaired energy expenditure (Lowell et al. 1993). Cardiac alterations in UCP-DTA mice have been interpreted as a result of the metabolic abnormalities in this mouse model associated with diet-induced obesity. However, the current awareness of the secretory role of BAT suggest that an additional explanation based on the impairment of the release of cardioprotective batokines (FGF21 or others) due to BAT ablation may account for cardiac alterations in the UCP-DTA mice.

\section{Myostatin and BAT-to-muscle signalling}

A relevant recent breakthrough in the identification of BAT-originated signalling to other tissues, that is, the endocrine role of BAT, is the recent identification of myostatin as a batokine that targets skeletal muscle. While several batokines have been reported to have the theoretical potential to target muscle, until recently no clear BAT-to-skeletal muscle signalling had been unequivocally identified. As a result of a recent study 
using experimental models of the targeted invalidation of the transcription factor IRF4 in BAT, Rosen and his collaborators (Kong et al. 2018) have identified skeletal muscle as an affected target and identified myostatin as a BAT-released factor mediating the BAT-to-muscle signalling.

Previous studies had already identified myostatin as a factor reciprocally associated with the extent of the thermogenic stimulus of BAT and the browning of WAT (Braga et al. 2013, Shan etal.2013), and a negative autocrine role of BAT activity had been proposed (Steculorum et al. 2016). Myostatin is a potent negative regulator of skeletal muscle growth (Rodriguez et al. 2014). Kong et al. have found that, under conditions of BAT inactivation such as treatment with thermoneutral temperature, myostatin levels were increased and the exercise capacity of skeletal muscle was lowered. In contrast, experimental activation of BAT lowered the myostatin levels and favoured exercise performance. Surgical BAT ablation blunted these effects on the muscles. In summary, BAT appears to control skeletal muscle function through the secretion of myostatin.

\section{Immune cells, novel targets of batokines: the chemokine C-X-C motif chemokine ligand-14 (CXCL14) as a brown adipokine, and beyond}

In recent years, the importance of immune cells infiltrating brown and beige adipose tissues for their recruitment and activation in response to thermogenic requirements has been reported (Villarroya et al. 2018a,b). Multiple reports have indicated that the recruitment of alternatively activated macrophages to BAT and beige adipose tissue is positively associated with thermogenic activation; however, the mechanisms by which macrophages intervene in BAT and/or beige adipose tissue activation remain controversial. The chemokine CXCL14 has been identified as a brown adipokine that is released by brown adipocytes in response to noradrenergic stimulation and leads to the alternative activation and recruitment of macrophages (Cereijo et al. 2018). Moreover, the CXCL14 released by BAT appears to influence the recruitment of M2 macrophages to subcutaneous WAT, thus promoting browning. This role of CXCL14 highlights the capacity of batokines to target immune cells. In fact, it is also known that increased pro-inflammatory status of BAT and beige adipose tissues (including the recruitment of pro-inflammatory immune cells) is negatively associated with their thermogenic activity. There are indications that factors secreted by thermogenically active brown adipocytes are capable of targeting pro-inflammatory cells and limit local inflammation. It has been reported that brown adipocytes are able to dampen the inflammatory responses in macrophages (Dowal et al. 2017). We have confirmed that the conditioned medium from brown adipocyte cultures represses the pro-inflammatory activity of M1-activated macrophages (Cereijo et al. 2018, Campderros et al. 2019). However, identifying the molecular actors involved in the signalling from brown/ beige adipocyte to immune cells has unveiled a complex scenario. While CXCL14 accounts for the recruitment and activation of M2 macrophages, it does not appear to directly influence pro-inflammatory signalling in M1 polarised macrophages. We recently found that growthand-differentiation factor-15 (GDF15), however, is secreted by brown adipocytes and exerts anti-inflammatory effects on M1 macrophages. GDF15 is a member of the TGF $\beta$ family that is widely recognised as a systemic marker of multiple pathologies, from cardiovascular disease to cancer. Recently, GDF15 has been reported to exert anorexigenic effects through its action at the brainstem and to contribute towards controlling the energy balance (Breit et al. 2017, Tsai et al. 2018). GDF15 is intensely secreted by brown fat upon exposure to cold conditions and by brown adipocytes after noradrenergic stimulation (Verdeguer et al. 2015, Campderros et al. 2019) and exerts mostly autocrine effects on macrophages, tuning down their pro-inflammatory responses (Campderros et al. 2019). Whether these signalling capacities of brown adipokines towards immune cells are restricted to the local cross-talk between immune cells and adipocytes at adipose depots or have consequences on the systemic immune status of the organism are challenging possibilities that remain to be ascertained in further researches.

Intriguingly, for many years, the cytokine interleukin-6 (IL-6) has been known to be secreted by BAT in response to thermogenic stimuli (e.g. cold environment) and by brown adipocytes in response to noradrenaline (Burýsek \& Houstek 1997), and is in fact, one of the molecules considered to be a potential batokine. The importance of the IL- 6 secreted by BAT has been evidenced by studies that showed that BAT explants from IL-6-KO mice (devoid of the capacity to secrete IL-6) do not show the capacity of wild-type BAT explants to promote a healthy metabolic response in models of experimental transplantation in mice (Stanford et al. 2013). Recently, the secretion of IL-6 by beige adipocytes has been reported to have positive autocrine actions upon the browning process, at least in vitro (Kristóf et al. 2019). Although it has not been 
directly addressed, it is possible that the reported effects of IL-6-induced M2 macrophages activation (Mauer et al. 2014) may play a role in the positive effects of BATsecreted IL-6. The recognition of a differential role of IL-6 in the past few years, whereby it acts as a cytokine or as a myokine (Pal et al. 2014), is suggestive of the necessity to further explore the physiological role of IL- 6 when it is specifically released by the BAT.

\section{BMP8b, a key brown adipokine for the adaptive remodelling of brown and beige adipose tissues to thermogenic demands}

In 2012, BMP8b, a member of the Bmp protein family, was identified as a brown adipokine released by brown adipocytes in response to noradrenergically mediated thermogenic stimulus (Whittle et al. 2012). Further studies have confirmed that $B m p 8 b$ is among the most intensely upregulated genes in murine brown adipocytes exposed to thermogenic stimuli (Verdeguer et al. 2015, Quesada-López et al. 2016). In contrast with other members of the Bmp family, the role of BMP8b in brown adipocyte biology is not associated with the early stages of adipogenic differentiation but with the secretory activity of fully differentiated brown adipocytes. Whittle and his collaborators (2012) found that BMP8b sensitises the BAT to sympathetically mediated thermogenic activation of BAT and WAT browning. Bmp8b gene expression in brown adipocytes is responsive not only to noradrenergic actions, but also to non-sympathetic activators of BAT activity such as G-protein-coupled receptor 120-mediated polyunsaturated fatty acid signalling (Quesada-López et al. 2016) or oestrogens (Grefhorst et al. 2015). Recent research has clarified some of the actions of the BMP8b secreted by brown adipocytes and its important autocrine and paracrine roles. Pellegrinelli et al. (2018) have found that BMP8b indirectly promotes sympathetic innervation and the vascularisation of brown and beige adipose tissues when the thermogenic capacity of the tissues has to be increased. BMP8b induces the endogenous production of NRG4, another known brown adipokine (Rosell et al. 2014, Wang et al. 2014), that promotes the outgrowth and branching of sympathetic nerve axons. Moreover, the release of BMP8b enhances the vascularisation of the brown fat depot by the induction of angiogenic factors. Therefore, BMP8b appears to be a brown adipocytesecreted factor that promotes active brown and beige adipose tissue remodelling to increase thermogenic efficiency by directly and indirectly targeting innervation and vascularisation, in a manner reminiscent of that by which brown adipocyte-secreted CXCL14 targets immune cells. Moreover, in addition to paracrine actions, it has been shown that BMP8b acts centrally and, via AMPK inhibition in the ventromedial hypothalamus and the subsequent increase in orexin signalling, leads to the sympathetically mediated thermogenic activation of BAT and WAT (Whittle et al. 2012, Martins et al. 2016). Whether the BMP8b that exerts these central actions originates in the BAT or is locally produced is currently unknown.

\section{The identification of an increasing number of brown adipokines targeting the sympathetic nerve endings at the BAT}

The extent of innervation of sympathetic nerve endings is essential for the functionality and activity of BAT depots. The capacity of secreted factors from brown adipocytes to target nerve endings and promote their outgrowth as a part of the brown fat recruitment process are among the first recognised biological roles of the components of the brown adipocyte secretome. In 1994, M Nechad and her collaborators had reported that co-cultures of BAT explants with sympathetic ganglia induced neurite outgrowth by producing a neurotrophic factor (Néchad et al. 1994), which was identified to be nerve growth factor (NGF); this effect was found to be more intense in case of BAT explants obtained from thermogenically stimulated rodent models (newborn hamsters, cold-exposed rats). This role of NGF, as a batokine targeting sympathetic neurite outgrowth was further confirmed (Nisoli et al. 1996); however, in this report, an inverse correlation between the NGF release and the extent of thermogenic BAT activation was found.

Later, Rosell and her collaborators identified NRG4 as a neurite outgrowth-promoting brown adipokine, preferentially expressed in thermogenically stimulated brown adipocytes (Rosell et al. 2014). As mentioned earlier, NRG4 has recently been found to respond to the autocrine effects of brown adipocyte-secreted BMP8b. Notably, B M Spiegelman's team has recently identified a new brown adipocyte-secreted factor: the S100b protein, which promoted neurite outgrowth from sympathetic neurons at adipose depots (Zeng et al. 2019). S100b does not contain a signalling peptide corresponding to standard secretion pathway; this may have hampered its identification as a batokine in previous studies. Although Zeng et al. have not reported the effects of experimental thermogenic stimuli (cold, noradrenaline) on S100b secretion, examination of 


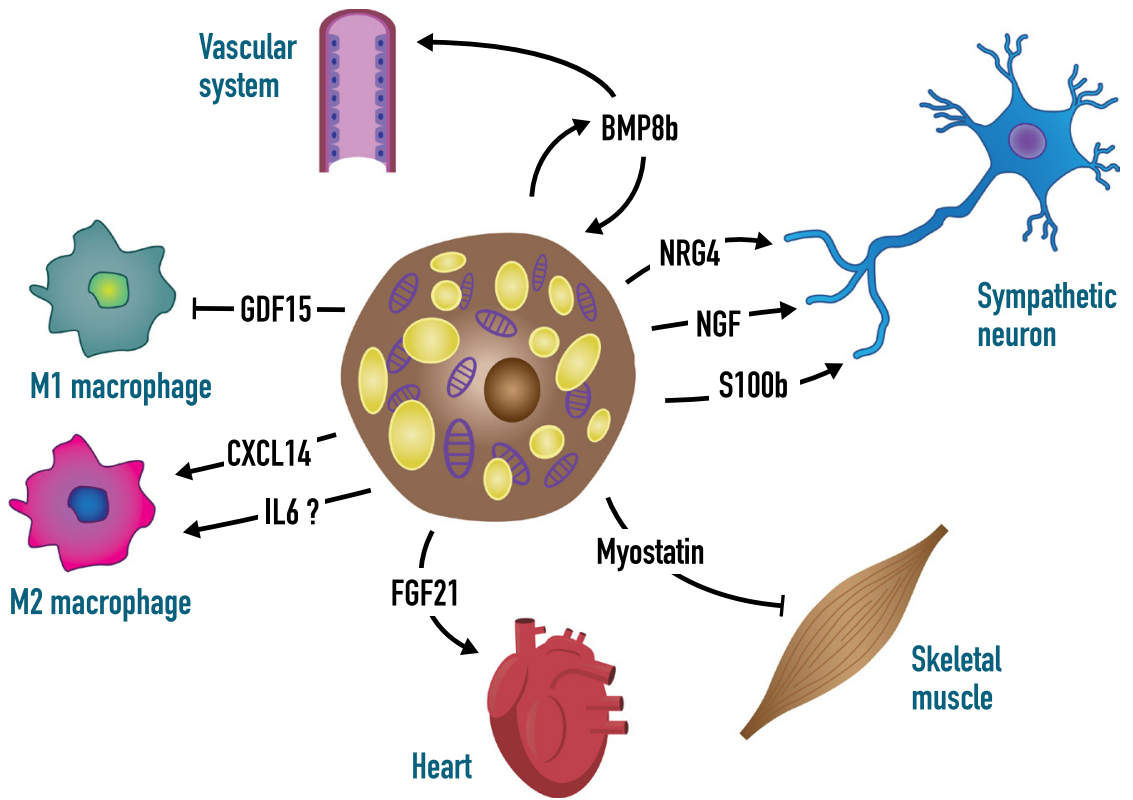

\section{Figure 2}

Representation of novel brown adipokines and their tissue targets. Bone morphogenetic protein-8b (BMP8b) targets vascular cells and, through its autocrine action on brown adipocytes, leads to the release of neuregulin-4 (NRG4) which promotes sympathetic neurite outgrowth. Nerve growth factor (NGF) and S100b protein are also secreted by brown adipocytes and target sympathetic nervous endings promoting innervation. C-X-C motif chemokine-14 (CXCL14) is a chemokine released by brown adipocytes; it promotes the recruitment of M2 macrophages, whereas brown adipocyte-secreted growth-anddifferentiation factor-15 (GDF15) inhibits the pro-inflammatory activity of M1 macrophages. The BAT-released fibroblast growth factor-21 (FGF21) targets the heart, favouring cardioprotective effects, whereas the level of BAT-secreted myostatin controls the performance of skeletal muscles. currently available transcriptomics databases (e.g. GEO GDS4850) indicate that acute cold exposure induces the expression of the $\mathrm{S} 100 \mathrm{~b}$ transcript in the BAT in mice; this is consistent with its role as a thermogenically induced batokine.

In summary, it appears that the secretome of thermogenically active brown adipocytes plays a key role in promoting the expansion of sympathetic nerve endings as a part of the recruitment of active BAT in response to environmental stimuli. NGF, NRG4, and S100b appear to be relevant components of the brown adipocyte secretome that mediate this action; however, we do not know whether there are other similar molecules, which are yet to be identified.

\section{Conclusions}

BAT is a secretory tissue producing brown adipokines, which exert autocrine, paracrine, and endocrine actions. In recent years, novel brown adipocyte-derived signalling molecules that have local actions and target vascular cells (e.g. BMP8b), macrophages (CXCL14, GDF15), and sympathetic nerve endings (NRG4, S100b), and contribute to the adaptive remodelling of BAT in response to thermogenic stimuli, have been identified. The capacity of BAT to serve as a source of endocrine factors has been recently reinforced by the recognition that BAT-released FGF21 targets the heart to exert cardioprotective effects. Moreover, BAT-secreted myostatin signals skeletal muscles to negatively regulate muscle performance (Fig. 2).
Recent proteomics-based analyses have unveiled novel components of the brown adipocyte secretome, such as members of the complement system and ECM components.

The extent of BAT activity, even in humans in which the BAT size is small, is associated with a healthy systemic metabolic profile. In addition to its intrinsic glucose- and lipid-oxidising capacity, the secretory role of BAT and its modulation according to its thermogenic activity may be related to this association. Further research is warranted into this aspect to comprehensively understand the BAT secretome, and the identity of brown adipokines and their targets and actions. The identification of brown adipokines and the characterisation of their effects is expected to enhance our knowledge regarding potential tools and targets useful in formulating intervention strategies for complex metabolic diseases such as obesity, diabetes, and cardiovascular diseases.

\section{Supplementary data}

This is linked to the online version of the paper at https://doi.org/10.1530/ JOE-19-0295.

\section{Declaration of interest}

The authors declare that there is no conflict of interest that could be perceived as prejudicing the impartiality of this review.

\section{Funding}

Supported by grants from Ministerio de Ciencia, Innovación y Universidades (SAF2017-85722) and Fondo de Investigaciones Sanitarias, Instituto de 
Salud Carlos III (PI17/00420), co-financed by the European Regional Development Fund (ERDF).

\section{References}

Ali Khan A, Hansson J, Weber P, Foehr S, Krijgsveld J, Herzig S \& Scheideler M 2018 Comparative secretome analyses of primary murine white and brown adipocytes reveal novel adipokines. Molecular and Cellular Proteomics 17 2358-2370. (https://doi. org/10.1074/mcp.RA118.000704)

Blüher M \& Mantzoros CS 2015 From leptin to other adipokines in health and disease: facts and expectations at the beginning of the 21st century. Metabolism: Clinical and Experimental 64 131-145. (https:// doi.org/10.1016/j.metabol.2014.10.016)

Braga M, Pervin S, Norris K, Bhasin S \& Singh R 2013 Inhibition of in vitro and in vivo brown fat differentiation program by myostatin. Obesity 21 1180-1188. (https://doi.org/10.1002/oby.20117)

Braga M, Reddy ST, Vergnes L, Pervin S, Grijalva V, Stout D, David J, Li X, Tomasian V, Reid CB, et al. 2014 Follistatin promotes adipocyte differentiation, browning, and energy metabolism. Journal of Lipid Research 55 375-384. (https://doi.org/10.1194/jlr.M039719)

Breit SN, Tsai VW-W \& Brown DA 2017 Targeting obesity and cachexia: identification of the GFRAL receptor-MIC-1/GDF15 pathway. Trends in Molecular Medicine 23 1065-1067. (https://doi.org/10.1016/j. molmed.2017.10.005)

Burýsek L \& Houstek J 1997 beta-Adrenergic stimulation of interleukin1alpha and interleukin- 6 expression in mouse brown adipocytes. FEBS Letters 411 83-86. (https://doi.org/10.1016/s0014-5793(97)00671-6)

Campbell DJ \& Habener JF 1987 Cellular localization of angiotensinogen gene expression in brown adipose tissue and mesentery: quantification of messenger ribonucleic acid abundance using hybridization in situ. Endocrinology 121 1616-1626. (https://doi. org/10.1210/endo-121-5-1616)

Campderros L, Moure R, Cairó M, Gavaldà-Navarro A, Quesada-López T, Cereijo R, Giralt M, Villarroya J \& Villarroya F 2019 Brown adipocytes secrete GDF15 in response to thermogenic activation. Obesity [epub]. (https://doi.org/10.1002/oby.22584)

Cannon B \& Nedergaard J 2004 Brown adipose tissue: function and physiological significance. Physiological Reviews 84 277-359. (https:// doi.org/10.1152/physrev.00015.2003)

Cereijo R, Gavaldà-Navarro A, Cairó M, Quesada-López T, Villarroya J, Morón-Ros S, Sánchez-Infantes D, Peyrou M, Iglesias R, Mampel T, et al. 2018 CXCL14, a brown adipokine that mediates brownfat-to-macrophage communication in thermogenic adaptation. Cell Metabolism 28 750.e6-763.e6. (https://doi.org/10.1016/j. cmet.2018.07.015)

Cook KS, Min HY, Johnson D, Chaplinsky RJ, Flier JS, Hunt CR \& Spiegelman BM 1987 Adipsin: a circulating serine protease homolog secreted by adipose tissue and sciatic nerve. Science 237 402-405. (https://doi.org/10.1126/science.3299705)

Crewe C, An YA \& Scherer PE 2017 The ominous triad of adipose tissue dysfunction: inflammation, fibrosis, and impaired angiogenesis. Journal of Clinical Investigation 127 74-82. (https://doi.org/10.1172/ JCI88883)

Datta R, Podolsky MJ \& Atabai K 2018 Fat fibrosis: friend or foe? JCI Insight 3 e122289. (https://doi.org/10.1172/jci.insight.122289)

Deshmukh AS, Peijs L, Nielsen S, Bayarri-Olmos R, Larsen TJ, Jespersen NZ, Hattel H, Holst B, Garred P, Tang-Christensen M, et al. 2018 Proteomics-based comparative mapping of the human brown and white adipocyte secretome reveals EPDR1 as a novel batokine. bioRxiv 402867. (https://doi.org/10.1101/402867)

Dowal L, Parameswaran P, Phat S, Akella S, Majumdar ID, Ranjan J, Shah C, Mogre S, Guntur K, Thapa K, et al. 2017 Intrinsic properties of brown and white adipocytes have differential effects on macrophage inflammatory responses. Mediators of Inflammation 2017 1-11. (https://doi.org/10.1155/2017/9067049)

Duncan JG, Fong JL, Medeiros DM, Finck BN \& Kelly DP 2007 Insulinresistant heart exhibits a mitochondrial biogenic response driven by the peroxisome proliferator-activated receptor-alpha/PGC-1alpha gene regulatory pathway. Circulation 115 909-917. (https://doi. org/10.1161/CIRCULATIONAHA.106.662296)

Grefhorst A, van den Beukel JC, van Houten EL, Steenbergen J, Visser JA \& Themmen AP 2015 Estrogens increase expression of bone morphogenetic protein $8 \mathrm{~b}$ in brown adipose tissue of mice. Biology of Sex Differences 6 7. (https://doi.org/10.1186/s13293-015-0025-y)

Hansen IR 2014 The secretome of brown adipose tissue. Doctoral thesis, Stockholm University, Stockholm, Sweden. (available at: https://www. diva-portal.org/smash/get/diva2:714076/FULLTEXT01.pdf)

Hansen IR, Jansson KM, Cannon B \& Nedergaard J 2014 Contrasting effects of cold acclimation versus obesogenic diets on chemerin gene expression in brown and Brite adipose tissues. Biochimica et Biophysica Acta 1841 1691-1699. (https://doi.org/10.1016/j. bbalip.2014.09.003)

Hondares E, Iglesias R, Giralt A, Gonzalez FJ, Giralt M, Mampel T \& Villarroya F 2011 Thermogenic activation induces FGF21 expression and release in brown adipose tissue. Journal of Biological Chemistry $\mathbf{2 8 6}$ 12983-12990. (https://doi.org/10.1074/jbc.M110.215889)

Hui X, Gu P, Zhang J, Nie T, Pan Y, Wu D, Feng T, Zhong C, Wang Y, Lam KS, et al. 2015 Adiponectin enhances cold-induced browning of subcutaneous adipose tissue via promoting M2 macrophage proliferation. Cell Metabolism 22 279-290. (https://doi.org/10.1016/j. cmet.2015.06.004)

Ilkun O, Wilde N, Tuinei J, Pires KM, Zhu Y, Bugger H, Soto J, Wayment B, Olsen C, Litwin SE, et al. 2015 Antioxidant treatment normalizes mitochondrial energetics and myocardial insulin sensitivity independently of changes in systemic metabolic homeostasis in a mouse model of the metabolic syndrome. Journal of Molecular and Cellular Cardiology 85 104-116. (https://doi.org/10.1016/j. yjmcc.2015.05.012)

Keipert S, Kutschke M, Lamp D, Brachthäuser L, Neff F, Meyer CW, Oelkrug R, Kharitonenkov A \& Jastroch M 2015 Genetic disruption of uncoupling protein 1 in mice renders brown adipose tissue a significant source of FGF21 secretion. Molecular Metabolism 4 537-542. (https://doi.org/10.1016/j.molmet.2015.04.006)

Kong X, Yao T, Zhou P, Kazak L, Tenen D, Lyubetskaya A, Dawes BA, Tsai L, Kahn BB, Spiegelman BM, et al. 2018 Brown adipose tissue controls skeletal muscle function via the secretion of myostatin. Cell Metabolism 28 631.e3-643.e3. (https://doi.org/10.1016/j. cmet.2018.07.004)

Kristóf E, Klusóczki Á, Veress R, Shaw A, Combi ZS, Varga K, Győry F, Balajthy Z, Bai P, Bacso Z, et al. 2019 Interleukin-6 released from differentiating human beige adipocytes improves browning. Experimental Cell Research 377 47-55. (https://doi.org/10.1016/j. yexcr.2019.02.015)

Lee MW, Lee M \& Oh KJ 2019 Adipose tissue-derived signatures for obesity and Type 2 diabetes: adipokines, batokines and microRNAs. Journal of Clinical Medicine E854 854. (https://doi.org/10.3390/ jcm8060854)

Lowell BB, Susulic V, Hamann A, Lawitts JA, Himms-Hagen J, Boyer BB, Kozak LP \& Flier JS 1993 Development of obesity in transgenic mice after genetic ablation of brown adipose tissue. Nature 366742 . (https://doi.org/10.1038/366740a0)

Lynes MD, Leiria LO, Lundh M, Bartelt A, Shamsi F, Huang TL, Takahashi H, Hirshman MF, Schlein C, Lee A, et al. 2017 The coldinduced lipokine 12,13-diHOME promotes fatty acid transport into brown adipose tissue. Nature Medicine 23 631-637. (https://doi. org/10.1038/nm.4297)

Martins L, Seoane-Collazo P, Contreras C, González-García I, MartínezSánchez N, González F, Zalvide J, Gallego R, Diéguez C, Nogueiras R, et al. 2016 A functional link between AMPK and orexin mediates https://joe.bioscientifica.com https://doi.org/10.1530/JOE-19-0295
(C) 2019 Society for Endocrinology Published by Bioscientifica Ltd.
Printed in Great Britain 
the effect of BMP8B on energy balance. Cell Reports 16 2231-2242. (https://doi.org/10.1016/j.celrep.2016.07.045)

Mauer J, Chaurasia B, Goldau J, Vogt MC, Ruud J, Nguyen KD, Theurich S, Hausen AC, Schmitz J, Brönneke HS, et al. 2014 Signaling by IL- 6 promotes alternative activation of macrophages to limit endotoxemia and obesity-associated resistance to insulin. Nature Immunology 15 423-430. (https://doi.org/10.1038/ni.2865)

Néchad M, Ruka E \& Thibault J 1994 Production of nerve growth factor by brown fat in culture: relation with the in vivo developmental stage of the tissue. Comparative Biochemistry and Physiology: Comparative Physiology 107 381-388. (https://doi.org/10.1016/03009629(94)90396-4)

Nisoli E, Tonello C, Benarese M, Liberini P \& Carruba MO 1996 Expression of nerve growth factor in brown adipose tissue: implications for thermogenesis and obesity. Endocrinology 137 495-503. (https://doi.org/10.1210/endo.137.2.8593794)

Pal M, Febbraio MA \& Whitham M 2014 From cytokine to myokine: the emerging role of interleukin-6 in metabolic regulation. Immunology and Cell Biology 92 331-339 (https://doi.org/10.1038/icb.2014.16)

Pellegrinelli V, Peirce VJ, Howard L, Virtue S, Türei D, Senzacqua M, Frontini A, Dalley JW, Horton AR, Bidault G, et al. 2018 Adipocytesecreted BMP8b mediates adrenergic-induced remodeling of the neuro-vascular network in adipose tissue. Nature Communications 9 4974. (https://doi.org/10.1038/s41467-018-07453-x)

Planavila A, Redondo I, Hondares E, Vinciguerra M, Munts C, Iglesias R, Gabrielli LA, Sitges M, Giralt M, van Bilsen M, et al. 2013 Fibroblast growth factor 21 protects against cardiac hypertrophy in mice. Nature Communications 4 2019. (https://doi.org/10.1038/ncomms3019)

Quesada-López T, Cereijo R, Turatsinze JV, Planavila A, Cairó M, GavaldàNavarro A, Peyrou M, Moure R, Iglesias R, Giralt M, et al. 2016 The lipid sensor GPR120 promotes brown fat activation and FGF21 release from adipocytes. Nature Communications 7 13479. (https://doi. org/10.1038/ncomms13479)

Rodriguez J, Vernus B, Chelh I, Cassar-Malek I, Gabillard JC, Hadj Sassi A, Seiliez I, Picard B \& Bonnieu A 2014 Myostatin and the skeletal muscle atrophy and hypertrophy signaling pathways. Cellular and Molecular Life Sciences 71 4361-4371 (https://doi.org/10.1007/s00018014-1689-x)

Rosell M, Kaforou M, Frontini A, Okolo A, Chan YW, Nikolopoulou E, Millership S, Fenech ME, MacIntyre D, Turner JO, et al. 2014 Brown and white adipose tissues: intrinsic differences in gene expression and response to cold exposure in mice. American Journal of Physiology: Endocrinology and Metabolism 306 E945-E964. (https://doi. org/10.1152/ajpendo.00473.2013)

Ruan CC, Kong LR, Chen XH, Ma Y, Pan XX, Zhang ZB \& Gao PJ 2018 $\mathrm{A} 2 \mathrm{~A}$ receptor activation attenuates hypertensive cardiac remodeling via promoting brown adipose tissue-derived FGF21. Cell Metabolism 28 476.e5-489.e5. (https://doi.org/10.1016/i.cmet.2018.06.013)

Shan T, Liang X, Bi P \& Kuang S 2013 Myostatin knockout drives browning of white adipose tissue through activating the AMPKPGC1 $\alpha$-Fndc5 pathway in muscle. FASEB Journal 27 1981-1989. (https://doi.org/10.1096/fj.12-225755)

Stanford KI, Middelbeek RJ, Townsend KL, An D, Nygaard EB, Hitchcox KM, Markan KR, Nakano K, Hirshman MF, Tseng YH, et al. 2013 Brown adipose tissue regulates glucose homeostasis and insulin sensitivity. Journal of Clinical Investigation 123 215-223. (https://doi. org/10.1172/JCI62308)

Steculorum SM, Ruud J, Karakasilioti I, Backes H, Engström Ruud L, Timper K, Hess ME, Tsaousidou E, Mauer J, Vogt MC, et al. 2016 AgRP neurons control systemic insulin sensitivity via myostatin expression in brown adipose tissue. Cell 165 125-138. (https://doi.org/10.1016/j. cell.2016.02.044)

Thomou T, Mori MA, Dreyfuss JM, Konishi M, Sakaguchi M, Wolfrum C, Rao TN, Winnay JN, Garcia-Martin R, Grinspoon SK, et al. 2017 Adipose-derived circulating miRNAs regulate gene expression in other tissues. Nature $\mathbf{5 4 2}$ 450-455. (https://doi.org/10.1038/nature21365)

Thoonen R, Ernande L, Cheng J, Nagasaka Y, Yao V, Miranda-Bezerra A, Chen C, Chao W, Panagia M, Sosnovik DE, et al. 2015 Functional brown adipose tissue limits cardiomyocyte injury and adverse remodeling in catecholamine-induced cardiomyopathy. Journal of Molecular and Cellular Cardiology 84 202-211. (https://doi. org/10.1016/j.yjmcc.2015.05.002)

Tsai VWW, Husaini Y, Sainsbury A, Brown DA \& Breit SN 2018 The MIC-1/GDF15-GFRAL pathway in energy homeostasis: implications for obesity, cachexia, and other associated diseases. Cell Metabolism 28 353-368 (https://doi.org/10.1016/j.cmet.2018.07.018)

Verdeguer F, Soustek MS, Hatting M, Blättler SM, McDonald D, Barrow JJ \& Puigserver P 2015 Brown adipose YY1 2015 deficiency activates expression of secreted proteins linked to energy expenditure and prevents diet-induced obesity. Molecular and Cellular Biology 36 184-196. (https://doi.org/10.1128/MCB.00722-15)

Villarroya F \& Giralt M 2015 The beneficial effects of brown fat transplantation: further evidence of an endocrine role of brown adipose tissue. Endocrinology 156 2368-2370. (https://doi. org/10.1210/en.2015-1423)

Villarroya F, Cereijo R, Villarroya J \& Giralt M 2017a Brown adipose tissue as a secretory organ. Nature Reviews: Endocrinology 13 26-35 35. (https://doi.org/10.1038/nrendo.2016.136)

Villarroya F, Gavaldà-Navarro A, Peyrou M, Villarroya J \& Giralt M $2017 b$ The lives and times of brown adipokines. Trends in Endocrinology and Metabolism 28 855-867. (https://doi.org/10.1016/j. tem.2017.10.005)

Villarroya F, Cereijo R, Gavaldà-Navarro A, Villarroya J \& Giralt M $2018 a$ Inflammation of brown/beige adipose tissues in obesity and metabolic disease. Journal of Internal Medicine 284 492-504. (https:// doi.org/10.1111/joim.12803)

Villarroya F, Cereijo R, Villarroya J, Gavaldà-Navarro A \& Giralt M $2018 b$ Toward an understanding of how immune cells control brown and beige adipobiology. Cell Metabolism 27 954-961. (https://doi. org/10.1016/j.cmet.2018.04.006)

Villarroya J, Cereijo R, Giralt M \& Villarroya F 2019 Secretory proteome of brown adipocytes in response to cAMP-mediated thermogenic activation. Frontiers in Physiology 1067 (https://doi.org/10.3389/ fphys.2019.00067)

Wang GX, Zhao XY, Meng ZX, Kern M, Dietrich A, Chen Z, Cozacov Z, Zhou D, Okunade AL, Su X, et al. 2014 The brown fat-enriched secreted factor Nrg4 preserves metabolic homeostasis through attenuation of hepatic lipogenesis. Nature Medicine 20 1436-1443. (https://doi.org/10.1038/nm.3713)

Whittle AJ, Carobbio S, Martins L, Slawik M, Hondares E, Vázquez MJ, Morgan D, Csikasz RI, Gallego R, Rodriguez-Cuenca S, et al. 2012 BMP8B increases brown adipose tissue thermogenesis through both central and peripheral actions. Cell 149 871-885. (https://doi. org/10.1016/j.cell.2012.02.066)

Zeng X, Ye M, Resch JM, Jedrychowski MP, Hu B, Lowell BB, Ginty DD \& Spiegelman BM 2019 Innervation of thermogenic adipose tissue

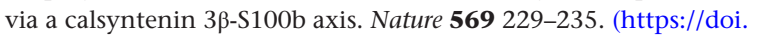
org/10.1038/s41586-019-1156-9)

Received in final form 12 August 2019

Accepted 16 August 2019

Accepted Preprint published online 16 August 2019 https://joe.bioscientifica.com https://doi.org/10.1530/JOE-19-0295 (c) 2019 Society for Endocrinology Published by Bioscientifica Ltd.
Printed in Great Britain 